\title{
General Rules for Optical Activity in Light Scattering of Plasmonic Nanostructures
}

Shu Gao, Junxiu Liu and Weihua Zhang*

College of Engineering and Applied Sciences, National Laboratory of Solid State Microstructures, and Collaborative Innovation Center of Advanced Microstructures, Nanjing University, Nanjing 210093, China

*Correspondence to: Weihua Zhang

Email: zwh@nju.edu.cn

\section{Operators for the electric and magnetic dipole moments}

In the main text, the electric dipole moment operator is written in a matrix form,

$$
\hat{\mathbf{P}}=\frac{\varepsilon_{0}}{s}\left[\begin{array}{lll}
1 & 0 & 0 \\
0 & 1 & 0 \\
0 & 0 & 1
\end{array}\right]
$$

This definition is equivalent to the conventional definition

$$
\mathbf{P}=\int_{\text {scatter }} \mathbf{p}(\mathbf{r}) d \mathbf{r}=\varepsilon_{0} / s \int_{\text {scatter }} \mathbf{E}(\mathbf{r}) d \mathbf{r}
$$


The proof is following. From eq. s1, the $i$ th component of $\mathbf{P}$ can be written as an inner product,

$$
P_{i}=\left\langle\mathbf{e}_{i}\left|\hat{P}_{i}\right| \mathbf{E}\right\rangle=\varepsilon_{0} / s \int_{\text {scatter }} \mathbf{e}_{i} \cdot\left[\begin{array}{ccc}
1 & 0 & 0 \\
0 & 1 & 0 \\
0 & 0 & 1
\end{array}\right]\left[\begin{array}{l}
E_{x} \\
E_{y} \\
E_{z}
\end{array}\right] d \mathbf{r}=\mathbf{e}_{i} \cdot\left(\varepsilon_{0} / s \int_{\text {scatter }} \mathbf{E}_{i}(\mathbf{r}) d \mathbf{r}\right) .
$$

This is exactly the $i$ th component of $\mathbf{P}$ in eq. $\mathrm{s} 2$.

In addition, in eq. s1, $\hat{\mathbf{P}}$ is real and diagonal, therefore, it is a Hermitian operator.

We can verify the definition of the operator of magnetic dipole moment in a similar way. In this work, the magnetic dipole moment operators are written as

$$
\hat{M}_{x}=\frac{i \omega \varepsilon_{0}}{s}\left[\begin{array}{ccc}
0 & -z & y \\
z & 0 & 0 \\
-y & 0 & 0
\end{array}\right], \hat{M}_{y}=\frac{i \omega \varepsilon_{0}}{s}\left[\begin{array}{ccc}
0 & -z & 0 \\
z & 0 & -x \\
0 & x & 0
\end{array}\right], \hat{M}_{z}=\frac{i \omega \varepsilon_{0}}{s}\left[\begin{array}{ccc}
0 & 0 & y \\
0 & 0 & -x \\
-y & x & 0
\end{array}\right]
$$

Here, we take the $x$ component as an example for the verification. From eq. s3, we have

$$
M_{n, x}=\left\langle\mathbf{e}_{x}\left|\hat{M}_{x}\right| \mathbf{E}_{n}\right\rangle=\frac{i \omega \varepsilon_{0}}{s} \int\left(y E_{n, z}-z E_{n, y}\right) d \mathbf{r}
$$

This is same as the conventional definition $\mathbf{M}=\int \mathbf{r} \times \mathbf{j}(\mathbf{r}) d \mathbf{r}$, in which

$$
M_{n, x}=\mathbf{e}_{x} \cdot \int \mathbf{r} \times \mathbf{j}_{n}(\mathbf{r}) d \mathbf{r}=-i \omega \varepsilon_{0} / s \int \mathbf{r} \times \mathbf{E} n(\mathbf{r}) d \mathbf{r}=\frac{i \omega \varepsilon_{0}}{s} \int\left(y E_{n, z}-z E_{n, y}\right) d \mathbf{r}
$$

Therefore, the matrix definition by eq. $\mathrm{s} 3$ is equivalent to the conventional definition of the magnetic dipole moment.

Same as the electric dipole moment operator $\hat{\mathbf{P}}$, the magnetic dipole operator $\hat{M}_{i}$ is also Hermitian. For $\hat{M}_{x}$, we have

$$
\left(\hat{M}_{x}\right)^{+}=\left(\frac{i \omega \varepsilon_{0}}{s}\left[\begin{array}{ccc}
0 & z & -y \\
-z & 0 & 0 \\
y & 0 & 0
\end{array}\right]\right)^{*}=\frac{i \omega \varepsilon_{0}}{s}\left[\begin{array}{ccc}
0 & -z & y \\
z & 0 & 0 \\
-y & 0 & 0
\end{array}\right]=\hat{M}_{x}
$$

Similarly, we can prove $\left(\hat{M}_{y}\right)^{+}=\hat{M}_{y}$ and $\left(\hat{M}_{z}\right)^{+}=\hat{M}_{z}$. 
We can also prove that the electric dipole moment operator $\hat{\mathbf{P}}$ commutes with magnetic dipole moment operator $\hat{M}_{i}$. Here, we use $\hat{M}_{x}$ as an example, $\hat{\mathbf{P}} \hat{M}_{x}=\left(\frac{\varepsilon_{0}}{s}\left[\begin{array}{ccc}1 & 0 & 0 \\ 0 & 1 & 0 \\ 0 & 0 & 1\end{array}\right]\right)\left(\frac{i \omega \varepsilon_{0}}{s}\left[\begin{array}{ccc}0 & -z & y \\ z & 0 & 0 \\ -y & 0 & 0\end{array}\right]\right)=\left(\frac{i \omega \varepsilon_{0}}{s}\left[\begin{array}{ccc}0 & -z & y \\ z & 0 & 0 \\ -y & 0 & 0\end{array}\right]\right)\left(\frac{\varepsilon_{0}}{s}\left[\begin{array}{lll}1 & 0 & 0 \\ 0 & 1 & 0 \\ 0 & 0 & 1\end{array}\right]\right)=\hat{M}_{x} \hat{\mathbf{P}}$ Similarly, $\hat{\mathbf{P}} \hat{M}_{y}=\hat{M}_{y} \hat{\mathbf{P}}$ and $\hat{\mathbf{P}} \hat{M}_{z}=\hat{M}_{z} \hat{\mathbf{P}}$.

Since both $\hat{\mathbf{P}}$ and $\hat{M}_{i}$ are Hermitian and commute with each other, $\hat{\mathbf{P}} \hat{M}_{i}$ is also Hermitian. In addition, $\hat{\mathbf{P}}$ is real, and $\hat{\mathbf{P}}=\hat{\mathbf{P}}^{*}$. Therefore, $\hat{\mathbf{P}}^{*} \hat{M}_{i}=\hat{\mathbf{P}} \hat{M}_{i}$ is also a Hermitian operator, and average of vector $\hat{\mathbf{P}}^{*} \hat{M}_{i}$, as well as each of its element $\hat{P}_{j}^{*} \hat{M}_{i}$, is always real (i.e., $\left.\operatorname{Im}\left(\hat{P}_{j}^{*} \hat{M}_{i}\right)=0\right)$

\section{Symmetry and optical activity of plasmonic naonstructures}

It is known that lack of rotation-reflection axes (symmetry center, symmetry plane, $2 \mathrm{~m}$-fold rotation-reflection axes $(2 \mathrm{~m} \geq 4))$ is necessary for a nanostructure to exhibit optical activity. In the main text, we prove that the polarization rotational power, $G_{i i}$ and rotational strength $R_{i i}$ for each eigenmode is always equal to zero for structures which have an inversion center.

In this section, we will give the proof for the rest two important cases, structures with reflection planes and rotation-reflection axes $\left(\mathrm{S}_{2 m}, 2 m \geq 4\right)$.

$\underline{\text { Structure with a plane of symmetry: }}$

Here, we use the Cartesian coordinate system with its $z$ axis perpendicular to the reflection plane. After a reflection operation, electric fields transform in the following way, 


$$
\hat{\sigma}[\mathbf{E}(\mathbf{r})]=\left(\begin{array}{c}
E_{x}(x, y,-z) \\
E_{y}(x, y,-z) \\
-E_{z}(x, y,-z)
\end{array}\right)
$$

Consequently, for the density of magnetic dipole moment $\mathbf{m}(\mathbf{r})=\frac{-i \omega \varepsilon_{0}}{s} \mathbf{r} \times \mathbf{E}(\mathbf{r})$, we have

$$
\hat{\sigma}[\mathbf{m}(\mathbf{r})]=\frac{-i \omega \varepsilon_{0}}{s} \hat{\sigma}\left(\begin{array}{l}
x \\
y \\
z
\end{array}\right) \times \hat{\sigma}\left(\begin{array}{c}
E_{x}(x, y, z) \\
E_{y}(x, y, z) \\
E_{z}(x, y, z)
\end{array}\right)=\frac{-i \omega \varepsilon_{0}}{s}\left(\begin{array}{c}
-y E_{z}(x, y,-z)+z E_{y}(x, y,-z) \\
-z E_{x}(x, y,-z)+x E_{z}(x, y,-z) \\
x E_{y}(x, y,-z)-y E_{x}(x, y,-z)
\end{array}\right)
$$

Because of the symmetry of the structure, eigenmode $\mathbf{E}_{n}$ is the base of an irreducible representation of the group and fulfills relation

$$
\hat{\sigma}\left[\mathbf{E}_{n}(\mathbf{r})\right]= \pm \mathbf{E}_{n}(\mathbf{r})
$$

We can therefore categorize all the eigenmodes into two groups, the symmetric modes and antisymmetric modes, corresponding to the positive and negative sign in eq. 88 , respectively.

For symmetric modes, $\hat{\sigma}\left[\mathbf{E}_{n}(\mathbf{r})\right]=\mathbf{E}_{n}(\mathbf{r})$, and in matrix form, it becomes

$$
\hat{\sigma}\left[\mathbf{E}_{n}(\mathbf{r})\right]=\left(\begin{array}{c}
E_{n, x}(x, y, z) \\
E_{n, y}(x, y, z) \\
E_{n, z}(x, y, z)
\end{array}\right)
$$

Inserting eq. s6 into eq. s9, we have

$$
\begin{aligned}
& E_{n, x}(x, y,-z)=E_{n, x}(x, y, z) \\
& E_{n, y}(x, y,-z)=E_{n, y}(x, y, z)
\end{aligned}
$$

and 


$$
E_{n, z}(x, y,-z)=-E_{n, z}(x, y, z)
$$

From eq. s11 and s2, we have

$$
P_{n, z}=0
$$

From eq. s7, we have

$$
m_{x}(x, y,-z)=\frac{-i \omega \varepsilon_{0}}{s}\left(y E_{z}(x, y,-z)+z E_{y}(x, y,-z)\right)
$$

Insert eq. s10 and s11 into eq. s13,

$$
m_{n, x}(x, y,-z)=\frac{-i \omega \varepsilon_{0}}{s}\left(-y E_{n, z}(x, y, z)+z E_{n, y}(x, y, z)\right)=-m_{n, x}(x, y, z)
$$

Similarly, for the $y$ component,

$$
m_{n, y}(x, y,-z)=-m_{n, y}(x, y, z)
$$

From eq. s14, we know $M_{x}=M_{y}=0$. Together with eq. s12, we have $R_{n, i i}=0$.

In the case of an antisymmetric mode, $\hat{\sigma}\left[\mathbf{E}_{n}(\mathbf{r})\right]=-\mathbf{E}_{n}(\mathbf{r})$. We can prove $P_{x}=P_{y}=M_{z}=0$, and consequently $R_{n, i i}=0$ in a similar way.

$\underline{\text { Structure with a } 2 m \text {-fold rotation-reflection axis }(2 \mathrm{~m}} \geq \underline{4)}$ :

In this part, we exam the remaining case, structures with rotation-reflection axes $S_{2 m}(2 m \geq 4)$. Since the structure is unchanged after $\mathrm{S}_{2 \mathrm{~m}}$ operation, $\hat{S}_{2 m} \mathbf{E}_{n}(\mathbf{r})$ is also an eigenmode of $\hat{\mathbf{G}}$ and consequently, we have

$$
\hat{S}_{2 m}\left[\mathbf{E}_{n}(\mathbf{r})\right]= \pm \mathbf{E}_{n}(\mathbf{r})
$$


If $m$ is odd, $\left(\hat{S}_{2 m}\right)^{m}$ equals to the inversion operator $\hat{I}$ and the structure is achiral. If $m$ is even, $\left(\hat{S}_{2 m}\right)^{m}=\hat{C}_{2}$. Together with eq. s15, we obtain

$$
\hat{C}_{2}\left[\mathbf{E}_{n}(\mathbf{r})\right]=\left(\hat{S}_{2 m}\right)^{m}\left[\mathbf{E}_{n}(\mathbf{r})\right]=\mathbf{E}_{n}(\mathbf{r})
$$

Assuming that the $\mathrm{z}$ axis is along the rotation-reflection axis, eq. $\mathrm{s} 16$ can be written as:

$$
\hat{C}_{2}\left[\mathbf{E}_{n}(\mathbf{r})\right]=\left(\begin{array}{c}
-E_{n, x}(-x,-y, z) \\
-E_{n, y}(-x,-y, z) \\
E_{n, z}(-x,-y, z)
\end{array}\right)=\left(\begin{array}{c}
E_{n, x}(x, y, z) \\
E_{n, y}(x, y, z) \\
E_{n, z}(x, y, z)
\end{array}\right)
$$

From eq. s17, we learn that, for any position $(x, y, z)$ in the $\mathrm{x}>0$ half space, there exists a point ($x,-y, z)$ in the $\mathrm{x}<0$ half space where

$$
\begin{aligned}
& E_{n, x}(x, y, z)=-E_{n, x}(-x,-y, z) \\
& E_{n, y}(x, y, z)=-E_{n, y}(-x,-y, z)
\end{aligned}
$$

Therefore,

$$
P_{x}=P_{y}=0
$$

Now, let us check the $z$ component of the electric and magnetic dipole. We know

$$
\hat{S}_{2 m}\left[\mathbf{E}_{n}(\mathbf{r})\right]=\left(\begin{array}{c}
E_{n, x}\left(x^{\prime}, y^{\prime}, z^{\prime}\right) \cos \theta_{2 m}-E_{n, y}\left(x^{\prime}, y^{\prime}, z^{\prime}\right) \sin \theta_{2 m} \\
E_{n, x}\left(x^{\prime}, y^{\prime}, z^{\prime}\right) \sin \theta_{2 m}+E_{n, y}\left(x^{\prime}, y^{\prime}, z^{\prime}\right) \cos \theta_{2 m} \\
-E_{n, z}\left(x^{\prime}, y^{\prime}, z^{\prime}\right)
\end{array}\right)
$$

where $2 m \theta_{2 m}=2 \pi$, and 


$$
\begin{aligned}
& x^{\prime}=x \cos \theta_{2 m}+y \sin \theta_{2 m} \\
& y^{\prime}=x \cos \theta_{2 m}+y \sin \theta_{2 m} \\
& z^{\prime}=-z
\end{aligned}
$$

In the case of positive sign in eq. s15 (i.e., $\left.\hat{S}_{2 m}\left[\mathbf{E}_{n}(\mathbf{r})\right]=\mathbf{E}_{n}(\mathbf{r})\right)$, we insert eq. s20 into eq. s15, and find that the $z$ component fulfills relation

$$
-E_{n, z}\left(x^{\prime}, y^{\prime},-z\right)=E_{n, z}(x, y, z)
$$

Therefore, for any position $(x, y, z)$ in the $\mathrm{z}>0$ half space, there exists a point $\left(x^{\prime}, y^{\prime},-z\right)$ in the $\mathrm{z}$ $<0$ half space where $-E_{n, z}\left(x^{\prime}, y^{\prime},-z\right)=E_{n, z}(x, y, z)$. Thus, we have $P_{z}=0$.

In the case of $\hat{S}_{2 m}\left[\mathbf{E}_{n}(\mathbf{r})\right]=-\mathbf{E}_{n}(\mathbf{r})$, we have

$$
\hat{S}_{2 m}\left[\mathbf{E}_{n}(\mathbf{r})\right]=\left(\begin{array}{c}
E_{n, x}\left(x^{\prime}, y^{\prime},-z\right) \cos \theta_{2 m}-E_{n, y}\left(x^{\prime}, y^{\prime},-z\right) \sin \theta_{2 m} \\
E_{n, x}\left(x^{\prime}, y^{\prime},-z\right) \sin \theta_{2 m}+E_{n, y}\left(x^{\prime}, y^{\prime},-z\right) \cos \theta_{2 m} \\
-E_{n, z}\left(x^{\prime}, y^{\prime},-z\right)
\end{array}\right)=-\left(\begin{array}{c}
E_{n, x}(x, y, z) \\
E_{n, y}(x, y, z) \\
E_{n, z}(x, y, z)
\end{array}\right)
$$

Thus,

$$
\begin{aligned}
& E_{n, x}\left(x^{\prime}, y^{\prime},-z\right) \cos \theta_{2 m}-E_{n, x}\left(x^{\prime}, y^{\prime},-z\right) \sin \theta_{2 m}=-E_{n, x}(x, y, z) \\
& E_{n, x}\left(x^{\prime}, y^{\prime},-z\right) \sin \theta_{2 m}+E_{n, x}\left(x^{\prime}, y^{\prime},-z\right) \cos \theta_{2 m}=-E_{n, y}(x, y, z)
\end{aligned}
$$

We know that the $z$ component of magnetic dipole can be expressed as

$$
m_{z}(x, y, z)=\frac{-i \omega \varepsilon_{0}}{s}\left(x E_{y}(x, y, z)+y E_{x}(x, y, z)\right)
$$

Inserting eq. s23 into eq. s24, we have 


$$
\begin{aligned}
& m_{z}(x, y, z)=\frac{-i \omega \varepsilon_{0}}{s}\left(x E_{y}(x, y, z)+y E_{x}(x, y, z)\right) \\
& =-\frac{-i \omega \varepsilon_{0}}{s}\left(\begin{array}{ll}
x & y
\end{array}\right)\left(\begin{array}{l}
E_{n, x}\left(x^{\prime}, y^{\prime},-z\right) \cos \theta_{2 m}-E_{n, x}\left(x^{\prime}, y^{\prime},-z\right) \sin \theta_{2 m} \\
E_{n, x}\left(x^{\prime}, y^{\prime},-z\right) \sin \theta_{2 m}+E_{n, x}\left(x^{\prime}, y^{\prime},-z\right) \cos \theta_{2 m}
\end{array}\right) \\
& =-\frac{-i \omega \varepsilon_{0}}{s}\left(\begin{array}{ll}
x^{\prime} & y^{\prime}
\end{array}\right)\left(\begin{array}{l}
E_{n, x}\left(x^{\prime}, y^{\prime},-z\right) \\
E_{n, x}\left(x^{\prime}, y^{\prime},-z\right)
\end{array}\right) \\
& =-\frac{-i \omega \varepsilon_{0}}{s}\left(x E_{y}\left(x^{\prime}, y^{\prime},-z\right)+y E_{x}\left(x^{\prime}, y^{\prime},-z\right)\right) \\
& =-m_{z}\left(x^{\prime}, y^{\prime},-z\right)
\end{aligned}
$$

Equation $\mathrm{s} 25$ indicates that, for each point $(x, y, z)$ in the $\mathrm{z}>0$ half space, there is a point $\left(x^{\prime}, y^{\prime},-\right.$

$z)$ where $m_{z}(x, y, z)=-m_{z}\left(x^{\prime}, y^{\prime},-z\right)$. Thus, $M_{z}=0$

From above, we know that $R_{n, i i}=0$ if a structure has a $2 m$-fold rotation-reflection axis, $(2 \mathrm{~m}$

$\geq 4)$. Therefore, the polarization rotation power $G_{i i}=\frac{1}{\varepsilon_{0}} \sum_{n} \frac{s^{2}}{s_{n}-s} \frac{1}{\omega} R_{n, i i}$ is always zero for plasmonic nanostructures with rotation-reflection axes.

\section{Perturbation theory}

Considering an achiral nanostructure, its scattered fields $\mathbf{E}$ satisfy the Lippmann-Schwinger equation, $\hat{\mathbf{G}}^{0} \mathbf{E}=s^{0} \mathbf{E}$. Here, $\hat{\mathbf{G}}^{0}$ is the Green's tensor operator, and $s_{0}$ is a material related parameter. It has a complete set of orthonormal eigenmode $\mathbf{E}_{n}^{0}$ associated with real eigenvalue $s_{n}^{0}$ $(n=1,2,3 \ldots)$.

When a dissymmetric part is added, the Lippmann-Schwinger equation becomes

$$
\mathbf{E}(\mathbf{r})=\mathbf{E}_{e x t}(\mathbf{r})+1 / s\left(\int_{V} d \mathbf{r}^{\prime} \mathbf{G}\left(\mathbf{r}, \mathbf{r}^{\prime}\right) \cdot \mathbf{E}\left(\mathbf{r}^{\prime}\right) k_{0}^{2}+\frac{\varepsilon_{N P}-\varepsilon_{b g}}{\varepsilon_{c a}-\varepsilon_{b g}} \int_{V^{\prime}} d r^{\prime} \mathbf{G}\left(\mathbf{r}, \mathbf{r}^{\prime}\right) \cdot \mathbf{E}\left(\mathbf{r}^{\prime}\right) k_{0}^{2}\right)
$$

where $V$ and $V$ 'is the volume of the original nanostructure and the additional dissymmetric part, respectively. Define

$$
\hat{\mathbf{G}}=\hat{\mathbf{G}}^{0}+\hat{\mathbf{G}}^{1}
$$


where $\hat{\mathbf{G}}^{0}$ is the Green's tensor operator for the unperturbed achiral part, and $\hat{\mathbf{G}}^{1}$ is operator associated with the dissymmetric part:

$$
\hat{\mathbf{G}}^{1} \mathbf{E}=\int_{V^{\prime}} d r^{\prime} k_{0}^{2} \mathbf{G}\left(\mathbf{r}, \mathbf{r}^{\prime}\right) \cdot \mathbf{E}\left(\mathbf{r}^{\prime}\right)
$$

Equation s26 becomes

$$
\hat{\mathbf{G}} \mathbf{E}=s \mathbf{E}
$$

If the volume of the dissymmetric part $V^{\prime}$ is much smaller than the volume of the original achiral nanostructure $V$, the first order perturbation can be applied and we have:

$$
\begin{gathered}
\mathbf{E}_{n} \approx \mathbf{E}_{n}{ }^{0}+\mathbf{E}_{n}{ }^{1} \\
s_{n} \approx \mathrm{S}_{n}{ }^{0}+\mathrm{S}_{n}{ }^{1}
\end{gathered}
$$

Inserting eq. s27, s30, and s31 into eq. s29, we obtain

$$
\mathbf{G}_{n}^{1} \mathbf{E}_{n}^{0}+\mathbf{G}_{n}^{0} \mathbf{E}_{n}^{1}=s_{n}^{1} \mathbf{E}_{n}^{0}+s_{n}^{0} \mathbf{E}_{n}^{1}
$$

Since $\mathbf{E}_{n}^{0}$ is complete, $\mathbf{E}_{n}{ }^{1}$ can be written as

$$
\mathbf{E}_{n}^{1}=\sum_{i} a_{i} \mathbf{E}_{i}^{0}
$$

Inserting eq. s33 into eq. s32 and multiplying with $\left\langle\mathbf{E}_{k}^{0}\right|$, eq. s32 becomes

$$
\left\langle\mathbf{E}_{k}^{0}\left|\mathbf{G}^{1}\right| \mathbf{E}_{n}^{0}\right\rangle+a_{k} s_{k}^{0}=s_{n}^{1} \delta_{n k}+s_{n}^{0} a_{k}
$$

If $k=n$, eq. $\mathrm{s} 34$ becomes

$$
s_{n}^{1}=\left\langle\mathbf{E}_{k}^{0}\left|\mathbf{G}^{1}\right| \mathbf{E}_{n}^{0}\right\rangle
$$

If $k \neq n$, eq. s34 becomes

$$
a_{k}=\frac{\left\langle\mathbf{E}_{k}^{0}\left|\mathbf{G}^{1}\right| \mathbf{E}_{n}^{0}\right\rangle}{s_{n}^{0}-s_{k}^{0}}
$$


Combining with eq. s36, eq. s33 can be written as

$$
\mathbf{E}_{n}^{1}=\sum_{k} \frac{\left\langle\mathbf{E}_{k}^{0}\left|\mathbf{G}^{1}\right| \mathbf{E}_{n}^{0}\right\rangle}{s_{n}^{0}-s_{k}^{0}} \mathbf{E}_{k}^{0}
$$

Here, $\left\langle\mathbf{E}_{k}^{0}\left|\mathbf{G}^{1}\right| \mathbf{E}_{n}^{0}\right\rangle=\int_{V} d \mathbf{r} \mathbf{E}_{l}^{0} \int_{V^{\prime}} d \mathbf{r}^{\prime} k^{2} \mathbf{G}\left(\mathbf{r}, \mathbf{r}^{\prime}\right) \mathbf{E}_{n}^{0}$. Since $V^{\prime}$ is very small, the electric field inside can be treated as a constant value and

$$
\left\langle\mathbf{E}_{k}^{0}\left|\mathbf{G}^{1}\right| \mathbf{E}_{n}^{0}\right\rangle=V^{\prime}\left(\int_{V} d \mathbf{r} \mathbf{E}_{l}^{0}(\mathbf{r}) \mathbf{G}\left(\mathbf{r}, \mathbf{r}^{\prime}\right)\right) \mathbf{E}_{n}^{0}\left(\mathbf{r}_{0}\right) \propto V^{\prime}
$$

where $\mathbf{r}_{0}$ is a point inside $V^{\prime}$. Inserting eq. s38 into eq. s37, we reach a simple linear relation

$$
\mathbf{E}_{n}^{1} \propto V^{\prime}
$$

\section{Optical rotational strength in the perturbation regime}

The optical rotation strength is proportional to the product of the electric dipole and magnetic dipole. For a nanostructure, the electric dipole associated with its eignemode $\mathbf{E}_{\mathrm{n}}$ can be written as $P_{n, i}=\left\langle\mathbf{e}_{i}\left|\hat{P}_{i}\right| \mathbf{E}_{n}\right\rangle$. Inserting eq. s30, we have

$$
P_{n, i}=\left\langle\mathbf{e}_{i}\left|\hat{P}_{i}\right| \mathbf{E}_{n}\right\rangle=\int_{V} d \mathbf{r} \frac{\varepsilon_{0}}{s} \mathbf{e}_{i} \cdot \mathbf{E}_{n}^{0}+\int_{V} d \mathbf{r} \frac{\varepsilon_{0}}{s} \mathbf{e}_{i} \cdot \mathbf{E}_{n}^{1}+\int_{V^{\prime}} d \mathbf{r} \frac{\varepsilon_{0}}{s} \mathbf{e}_{i} \cdot \mathbf{E}_{n}^{0}+\int_{V^{\prime}} d \mathbf{r} \frac{\varepsilon_{0}}{s} \mathbf{e}_{i} \cdot \mathbf{E}_{n}^{1}
$$

In the perturbation regime, $V^{\prime}<<V, \mathbf{E}_{n}^{1}<<\mathbf{E}_{n}^{0}$, and therefore, the last term on the right side of the equation is negligible, and eq. s40 becomes

$$
P_{n, i}=\int_{V} d \mathbf{r} \frac{\varepsilon_{0}}{S} \mathbf{e}_{i} \cdot \mathbf{E}_{n}^{0}+\int_{V} d \mathbf{r} \frac{\varepsilon_{0}}{S} \mathbf{e}_{i} \cdot \mathbf{E}_{n}^{1}+\int_{V^{\prime}} d \mathbf{r} \frac{\varepsilon_{0}}{S} \mathbf{e}_{i} \cdot \mathbf{E}_{n}^{0}=P_{n, i}^{0}+P_{n, i}^{1}
$$

Here,

$$
P_{n, i}{ }^{0}=\int_{V} d \mathbf{r} \frac{\varepsilon_{0}}{S} \mathbf{e}_{i} \cdot \mathbf{E}_{n, i}^{0}, \text { and } P_{n, i}{ }^{1}=\int_{V} d \mathbf{r} \frac{\varepsilon_{0}}{s} \mathbf{e}_{i} \cdot \mathbf{E}_{n}^{1}+\int_{V^{\prime}} d \mathbf{r} \frac{\varepsilon_{0}}{S} \mathbf{e}_{i} \cdot \mathbf{E}_{n}^{0}
$$


Since $V^{\prime}$ is small, $\mathbf{E}_{n}^{0}$ is approximately a constant. Hence,

$$
\int_{V^{\prime}} d \mathbf{r} \frac{\varepsilon_{0}}{S} \mathbf{e}_{i} \cdot \mathbf{E}_{n}^{0}=\frac{\varepsilon_{0}}{S} \mathbf{e}_{i} \cdot \mathbf{E}_{n}^{0} V^{\prime} \propto V^{\prime}
$$

In addition, from eq. s39 we know $\mathbf{E}_{n}^{1} \propto V^{\prime}$ and therefore

$$
\int_{V} d \mathbf{r} \frac{\varepsilon_{0}}{S} \mathbf{e}_{i} \cdot \mathbf{E}_{n}^{1} \propto V^{\prime}
$$

Inserting eq. s43, s44 into s42, we have

$$
P_{n, i}^{1}=\int_{V} d \mathbf{r} \frac{\varepsilon_{0}}{s} \mathbf{e}_{i} \cdot \mathbf{E}_{n}^{1}+\int_{V^{\prime}} d \mathbf{r} \frac{\varepsilon_{0}}{s} \mathbf{e}_{i} \cdot \mathbf{E}_{n}^{0} \propto V^{\prime}
$$

Similarly, the magnetic dipole of the system can be written as $M_{n, i}=M_{n, i}^{0}+M_{n, i}^{1}$ with $M_{n, i}{ }^{1} \propto V^{\prime}$

The optical rotational strength $R_{n, i i}$ of the perturbed system can be written as $R_{n, i i}=\operatorname{Im}\left(P_{n i}^{0}+P_{n i}^{1}\right)^{*}\left(M_{n i}^{0}+M_{n i}^{1}\right)$. Knowing that for the original achiral nanostructure $R_{n, i i}{ }^{0}=\operatorname{Im} P_{n i}^{0^{*}} M_{n i}^{0}=0, P_{n i}^{0} \gg>P_{n i}^{1}$ and $M_{n i}^{0}>>M_{n i}^{1}$, the optical rotational strength can be simplified as

$$
R_{n, i i}=\operatorname{Im}\left(P_{n i}^{1^{*}} M_{n i}^{0}+P_{n i}^{0^{*}} M_{n i}^{1}\right)
$$

Combining eq. s45, s46, and s47, we finally obtain a linear relation

$$
R_{n, i i} \propto V^{\prime}
$$

